\title{
AERODYNAMICS OF FLUE ORGAN PIPE VOICING
}

\author{
D. Steenbrugge ${ }^{1}$, P. De Baets ${ }^{2}$ \\ ${ }^{1}$ Hogeschool Gent, Belgium \\ ${ }^{2}$ Ghent University, Laboratory Soete, Belgium
}

\begin{abstract}
The objective of this paper is to investigate the possibility of giving useful interpretations of flue organ pipe voicing practices in terms of the aerodynamical and aeroacoustical behaviour of the pipes. An overview is first given of the current state of the knowledge on sound generation in flue instruments. After an introduction into the limited literature on voicing an scheme is presented as a possible framework to classify and characterize various voicing approaches. Use is made of dimensionless analysis to quantify the specific properties of voicing methods in terms of aerodynamic parameters rather than geometric data. It is concluded that such an analysis might be a useful tool to be able to better document and understand historic instruments and their genesis in order to better conserve them.
\end{abstract}

Keywords musical acoustics, aeroacoustics, aerodynamics, resonators, flue organ pipes, pipe organs, pipe voicing, organ building, conservation,

\section{INTRODUCTION}

Associating the term aerodynamics with an activity which always has been considered as the culprit of craftsmanship and artistry in organ building may seem a little respectless at first, yet it is a fact that organ pipes above all are aerodynamical systems. Particularly in flue organ pipes, and just like in all other flute instruments, essentially only the air inside is vibrating and the solid geometry 'merely' serves to exploit the instabilities inherent in the fluid so as to cause it to perform more or less periodic movements. As will be stressed in this paper however, the acoustic radiation, which is what the pipe is made for, turns out to be only a small side effect of these movements. This, together with the elusiveness of the vibrating medium, has obliged the instrument builders to develop empirical and highly individual approaches towards sound design typical and indispensable for the creation of true art. Interfering in this process by means of modern science is not going to make even better art, but opens other possibilities. Different kinds of art arise under the influence of modern science, for instance in sound design using current technologies, and it can benefit from results obtained by viewing traditional sound production using more recently obtained knowledge. Such is the case in sound synthesis based on physical models of traditional (acoustic) instruments. This paper however is intended to bring a contribution to another important application of modern knowledge, namely the conservation of art. Knowing how a work of art has been conceived and realised is an extremely important, although difficult to grasp aspect of art conservation. This is the very point where the individualistic nature of art has to face its ephemerality.

So the main argument in this paper will be that physics can be used as a tool in the analysis of the original instruments, with the goal to effectively describe and organize how their came into being, yet admittedly leaving the most subtle aspects to the expert. From a conservation point of view, this evolution is inevitable and throughout history evidence shows this kind of codification to be a necessary step in order to conserve at least some part of the original works. Books and music scores represent only a part of what the creator of their contents wanted to express, but they are a way of codification without which nothing at all would be left of his message after some time. Similarly, masterworks of all kinds deteriorate but if one can grasp and codify part of their genesis then at least part of them can survive.

The sciences of aerodynamics and aeroacoustics are relatively young, most of their fundaments having been developed from not much more than a century ago onwards. Accordingly it's not surprising that the art and craft of flue organ pipe voicing doesn't show any connection with them, not even in the great XIXth and XXth century 'theoretical' works on organ building [Töpfer 1888, Audsley 1905, Ellerhorst 1936]. The first contacts date from the 1960's were a number of physicists became interested in developing a scientific view on the drive mechanism of flue instruments. Since then a few research groups in the world have established themselves as leading the progress, but the main motivation has usually been the intention to build better physical models capable of reproducing naturally (mechanically) generated sound. Very little attention has been given to the potential applications in the field of conservation of musical instruments.

This paper will first give a very brief overview of current mainstream knowledge on the aerodynamics (and some acoustics) of flue instruments, thereby giving an idea of the basic operating principles. Next the stage is completely changed in order to give an idea of what has been published on the subject of flue organ pipe voicing. In the next part a scheme will be proposed as a possible framework within which to characterize 
voicing techniques. This framework is based on aerodynamic rather than on geometric parameters. Finally some proposals and examples are given on how traditional voicing rules can be expressed and organized in terms of this framework and its associated aerodynamic parameters.

As this text is meant, by its interdisciplinary character, to be readable by a relatively broad audience, attention is given more to a qualitative presentation of concepts rather than a mathematical treatment. Various experimental results reproduced here as illustrations have been obtained from an experimental flue pipe with precision controllable geometry, the complete description and preliminary measurements obtained with it are reported elsewhere [Steenbrugge 2009].

\section{AN OVERVIEW OF CURRENT KNOWLEDGE ON THE AERODYNAMICS OF FLUE INSTRUMENTS}

\subsection{General principles}

The mechanical operation of musical instruments is generally subdivided into a linear 'acoustic' part, essentially a resonator, and a non-linear part, which transforms a steady input flow of energy into more or less regular flow bursts [Mclntyre, Schumacher \& Woodhouse 1983]. These parts are interconnected and feedback loops can be identified which, under appropriate conditions, allow the system to perform periodic self-sustained oscillations. Based on this scheme the flue instrument is modelled on the one hand as a onedimensional air column subject to specific boundaries allowing certain, more or less harmonic, passive resonances to develop, and on the other hand as an air jet flow interacting with the acoustic field of the resonator. The air jet exits through a long straight flue and is therefore considered to be more or less twodimensional. This flue is generally positioned near one of the open ends of the air column. Consequently the air jet can exchange energy with the acoustic field and vice versa: the latter can strongly influence the behaviour of the inherently unstable air jet. In order for the air jet to produce flow bursts it needs a separating edge, the upper labium, placed downstream which will cause the air jet flow to be alternately directed inwards and outwards once the feedback loop enters a stable regime.

The energy exchange process within the pipe, which includes volume injection as well as turbulent mixing of air flows, is of the order of only a few percent and the remaining energy is further dissipated mostly through friction and thermal losses at the walls, so that finally little is left over as acoustic radiation energy [Fletcher \& Rossing 2005]. Despite this dissipation the mutual interaction between air jet and acoustic flow is strong enough to drive a positive feedback loop which can, under favourable circumstances, generate self-sustained oscillations. Furthermore, because of the relatively sharp impedance minima of the strongly coupled resonator, at which frequencies the pipe air flows can be particularly strong, the periodicity of the oscillations will be essentially determined by these air column resonances.

\subsection{The resonator}

The acoustic behaviour of wind-instrument bores, including various nearly-cylindrical profiles, has been fairly well understood since many decades. The acoustic properties of these bores, including their strong dependence on their cross-section, can be found in many classic acoustics texts [Pierce 1990] and will not be repeated here. In recorders the bore diameter is frequently adjusted in the voicing stage in order to optimize individual notes. In organ building however the bore diameter is normally kept the way it is made, except maybe at the (open or half-open) end, because considerable modifications are still possible at the mouth region knowing that the organ pipe must be optimized for only one frequency.

Because voicing the organ pipe mostly deals with making adjustments influencing the mouth end correction, which is much larger than a normal open end correction, this aspect of resonators will be looked at somewhat closer here.

For an unflanged pipe end it was calculated [Levine \& Schwinger 1948] at low frequencies the end correction is about $0.61 \mathrm{a}$, where $\mathrm{a}$ is the pipe radius, approximately varies as $0.6 \mathrm{a}-0.1 \mathrm{ka}^{2}$, where $\mathrm{k}$ is the wavenumber, as long as $\mathrm{ka}<4$, and essentially 0 when $\mathrm{ka}>4$. The end correction of the mouth region cannot be exactly calculated but approximate formulae exist, such as the well-known low-frequency formula [Ingerslev and Frobenius 1047] for a rectangular mouth cut in a cylindrical tube:

$$
L_{m}=\frac{2.3 a^{2}}{\sqrt{H W}}
$$

where $\mathrm{H}$ is the cut-up and $\mathrm{W}$ the width of the mouth. These formulae no longer apply if tuning slots, sleeves or ears are applied. 
The effect of the end corrections can be seen in the following measurement of the passive resonances of an open organ pipe, obtained by measuring the open end sound pressure while injecting white noise into the mouth:

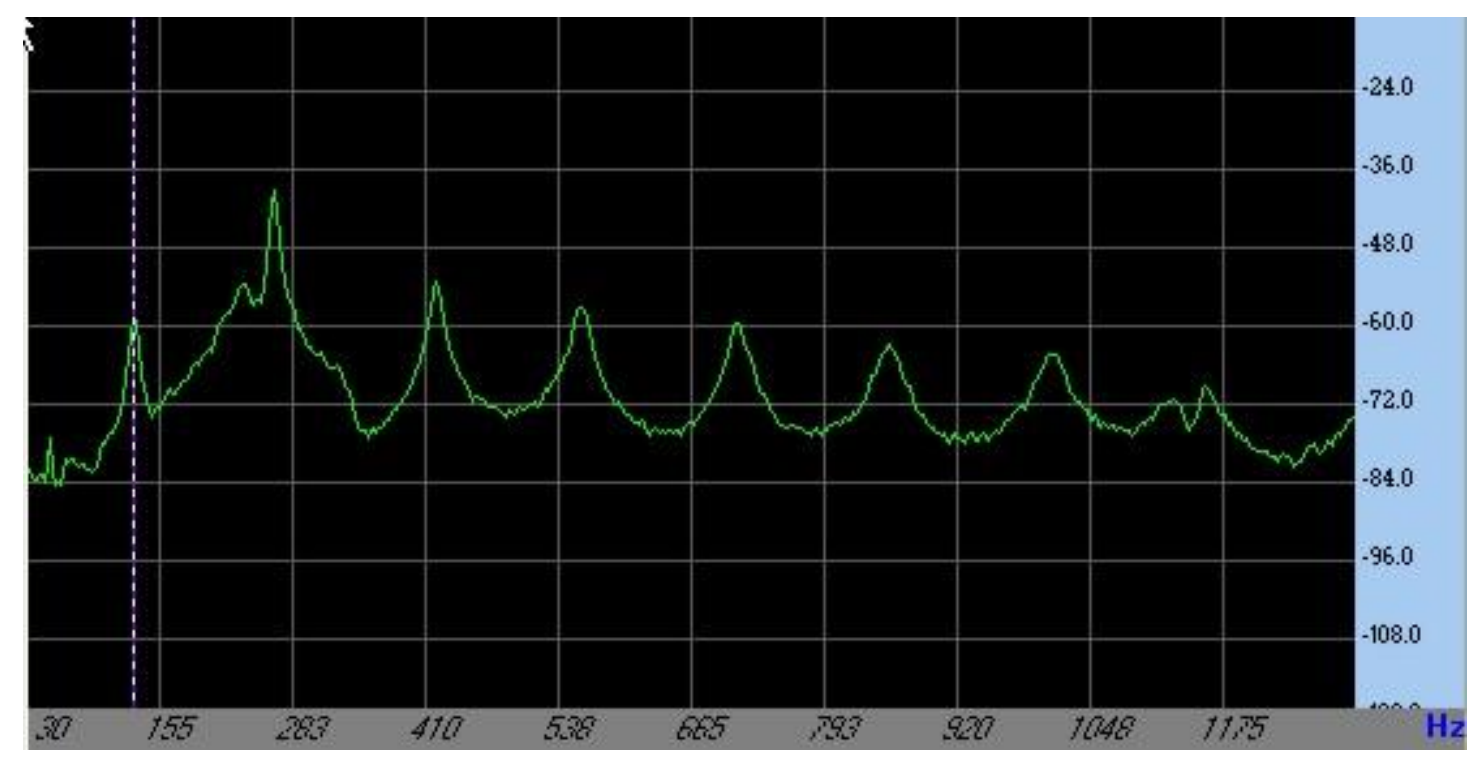

The cursor indicates the first passive resonance of $130 \mathrm{~Hz}$. Higher resonances are successively stretched and thus inharmonic.

The next measurement shows the open end spectrum of the same pipe blowing at $130 \mathrm{~Hz}$ :

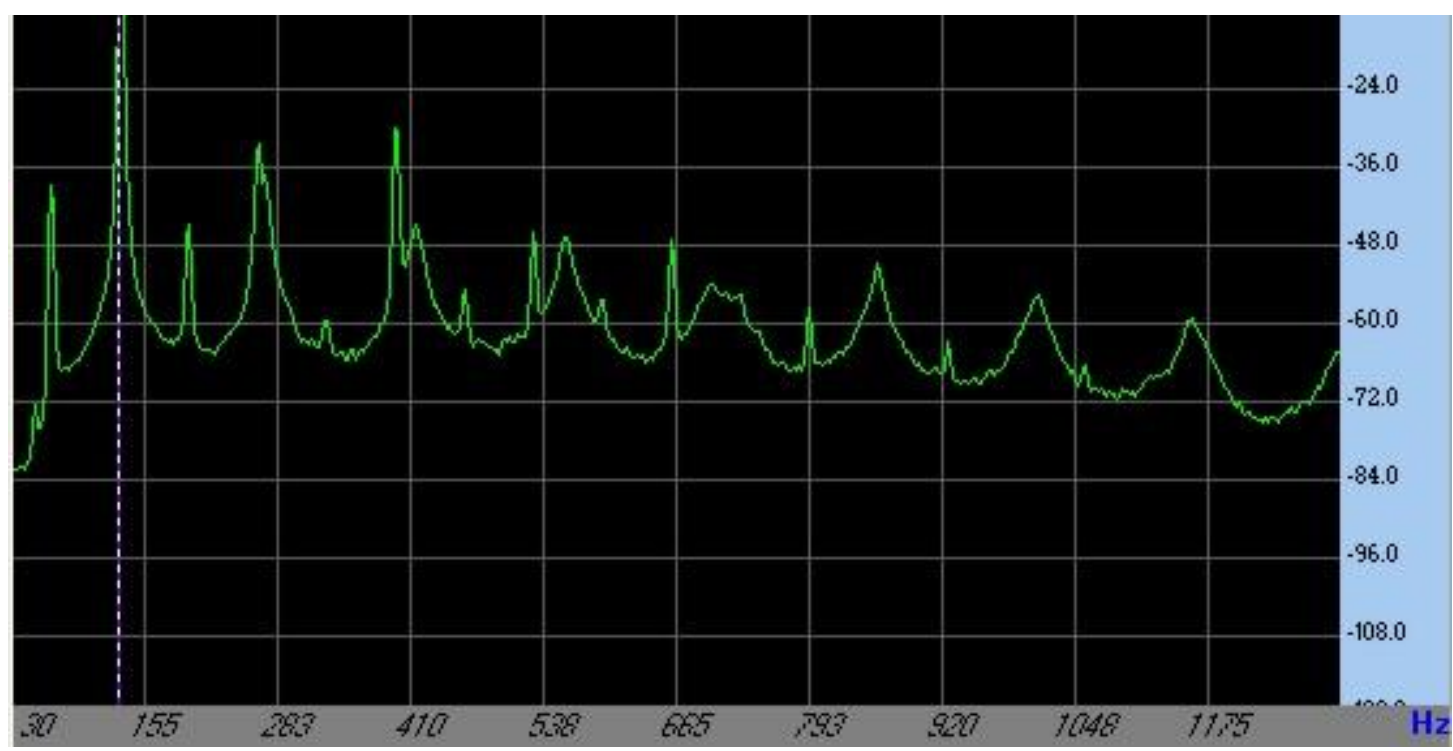

Two additional series of peaks are visible:

- Sharp peaks at the exact harmonic locations of the fundamental $130 \mathrm{~Hz}$

- Some sharp peaks as uneven harmonics of half the fundamental caused by reflection against the measuring microphone.

Those harmonics that coincide best with any of the passive resonances (in this case the first few) will sound stronger. The higher ones only coincide now and then.

The way the end correction voicing, especially of the mouth, influences the pipe resonances can be characterized by a dimensionless parameter $L_{m} / L$. For narrow-scaled pipes this value will be, considering (1) and typical proportions of the mouth, of order 0.05 , corresponding to the soft violinlike sound of this so- 
called string family. The other extreme two orders of magnitude larger is reached with the broad-scaled dull sounding flute pipes, where everything contributes to maximum inharmonicity.

\subsection{The air jet driven by the resonator}

Fluid jets have been extensively studied, including the particular case of the two-dimensional air jet subjected to an air cross-flow. Their behaviour is governed by the strong shear layers between the two flows and this behaviour turns out to be very difficult to treat analytically from the basic fluid dynamics laws. Therefore many approximate physical models have been developed applicable within the range of certain assumptions made.

A basic feature of the air jet in flue instruments is its intrinsic instability, which has as a consequence that a small disturbance imposed on the jet by the external flow (for instance the flow of an acoustic field) is not only propagated on the jet but also amplified. Both effects contribute much to the positive feedback loop mentioned before. A complete theory on exactly how the cross-flow influences the air jet remains to be developed [Fletcher 2001]. The most recent studies on this subject [Nolle 1998, Verge 1995, Fabre 2000] seem to favour, by the lack more physically consistent theories, a semi-empirical model describing the jet trajectory which assumes an exponential amplitude growth of any disturbances propagated along the jet, combined with a simple convection with the cross-flow, and satisfying the basic boundary condition that the jet exits a stationary flue. When the cross-flow velocity is assumed to be $v \cdot \cos (\omega t)$, where $t$ is the time, $\omega$ the radial frequency and $v$ the velocity amplitude, the displacement $y$ along the jet trajectory as a function of the distance $x$ from the flue along the direction towards the upper labium is:

$$
y(x)=v\left[\cos \omega t-\cosh \mu x \cdot \cos \left(\omega\left(t-\frac{x}{u}\right)\right)\right]
$$

where additionally:

$$
\begin{array}{ll}
u & \text { the phase velocity of the disturbances propagating on the jet } \\
\mu & \text { the exponential growing coefficient }
\end{array}
$$

The meaning of most of these parameters is clarified in the next figure, showing in profile the air jet issuing from the flue exit and heading towards the upper labium of the flue instrument, with the coupled resonator on the lower side.

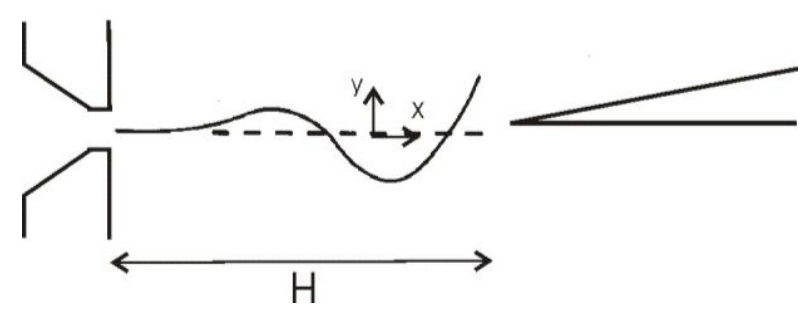

$\mathrm{U}$ depends in a complex way on the air jet flow velocity $\mathrm{V}$, which in turn depends on the air supply pressure P. As a first approximation it can be stated:

where:

$$
\begin{aligned}
& u \approx 1 / 2 V \\
& V \approx \sqrt{\frac{2 P}{\rho}} \quad \text { or } V \approx 13 \sqrt{P} \quad \text { if } \mathrm{P} \text { is expressed in } \mathrm{cm}_{2} \mathrm{O}
\end{aligned}
$$

$\rho$ is the air density

From (2) the disturbance displacement at the flue is seen to lead the acoustic velocity by $1 / 4$ of a period, and the disturbance travels the distance $\mathrm{H}$ over the jet from the flue to the upper labium, the cut-up, during the fraction $\mathrm{fH} / \mathrm{u}$ of a period.

The velocity profile of the jet depends on the windchannel. If there is a long windchannel like in recorders the the velocity profile will be that of a fully developed flow, in the other extreme, more likely in organ pipes, it will be more like a top-hat at the flue, but will rapidly develop into a lateral profile described by a sech ${ }^{2}$ in case of laminar flow, the so-called Bickley profile. Turbulent jets have a velocity behaviour that is even 
more complex, much more details on air jets in flue instruments can be found among others in [Nolle 1998, Segoufin 2001, Yoshikawa 1999].

\subsection{The resonator drive by the air jet}

As the air jet is driven by the acoustic flow from the resonator it is best placed at an admittance maximum. However, in order to drive the air in the resonator and deliver energy, the air jet needs a pressure to work against. An open end is an admittance maximum looking into the pipe, which is favourable for the first condition, and a real acoustic open end always presents a non-zero impedance to the acoustic flow, the end correction, so the pressure at the open end is not perfectly zero. Accordingly an open end is thus a suitable location for an air jet to interact with the resonator and this is found in all flue instruments.

The interaction of the jet with the air column in the pipe is a complex fluid dynamical phenomenon the details of which are not yet completely clear. The air jet flow injects volume but also contributes momentum, both contributions have a different dependency on $\mathrm{V}$ and imply different phase shifts. It turns out that in practice the volume contribution dominates [Fletcher\&Rossing 2005] and very roughly the jet drive can be seen, in a linear approach, as a 'current' source driving the resonator, with impedance $Z_{p}$, and the mouth, with impedance $Z_{\mathrm{m}}$, in parallel, so that:

$$
v=\frac{Z_{p}}{Z_{p}+Z_{m}} \cdot \frac{V \cdot S_{j}}{S_{m}}
$$

where:

$S_{j}$ is the (periodically varying) cross-section of the part of the air jet flowing into the resonator:

$$
S_{j}=\begin{aligned}
& V(y+b) \text { if } y<b, \text { where } b \text { is the jet half width at the upper labium } \\
& V 2 b \text { if } y>b
\end{aligned}
$$

$\mathrm{S}_{\mathrm{m}}$ is the surface of the mouth.

As an electrical network equivalent this can be represented as:

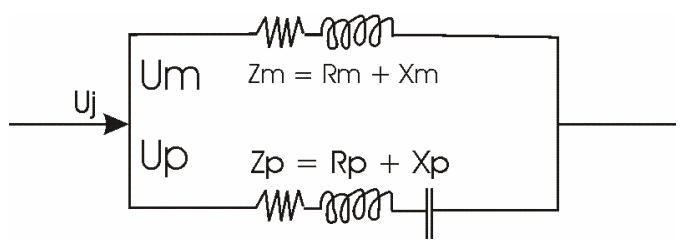

\subsection{Closing the feedback loop}

Clearly in (5) the acoustic field velocity $v$ leads the air jet flow VS in phase by $1 / 4$ of a period, so that in order for the complete feedback loop to be closed the disturbance transit time $\mathrm{fH} / \mathrm{u}$ must be $1 / 2$ of a period. Moreover, in an open end pipe the transit time for a pressure disturbance to travel from the mouth to the open end and back is of course also equal to this period, so that the, in reality double, feedback loop is closed. With a closed end pipe the pressure disturbance simply performs 4 transits through the pipe, being reflected once in the middle of the period at the open end. In practice the loop tolerates considerable deviations from these values because in normal operation the frequency is near a point of low $Z_{p}$, where the phase of $Z_{p}$ rapidly switches from $-\pi / 2$ to $\pi / 2$, and thus can considerable adjust the phase shift implied in (5). This explains why the frequency very slightly rises when the blowing pressure rises and vice versa.

The amplitudes reached in the steady loop is determined by the balance of energy supply and losses. Both increase with increasing amplitudes, but at a certain point the energy supply saturates as the air jet is fully blowing into the pipe.

\subsection{Harmonic generation:}

The complete feedback loop is mainly driven by the fundamental frequency of the sound, higher harmonics do not much intervene, rather they are generated at different places by various non-linearities. The air jet flow constitutes the main nonlinearity of the feedback loop [Fletcher \& Douglas 1980], due to the velocity profile and to flow saturation when the jet displacement at the upper labium becomes so large that it completely flows into one side for an extended time. When the air jet flow is perfectly symmetric around the upper labium the spectrum of the flow into the resonator will be mainly driven by uneven harmonics of the fundamental. Other values of asymmetry, which can be obtained by giving the air jet a different orientation 
or displacing the upper labium position with respect to the air jet mid-plane, will suppress various other multiples. A simulation of this phenomenon [Fletcher \& Douglas 1980] shows regular minima and maxima for the various harmonics, the even harmonics having a minimum at the symmetry axis.

The next figure shows this behaviour as it was observed in the experimental flue pipe when laterally displacing the upper labium:

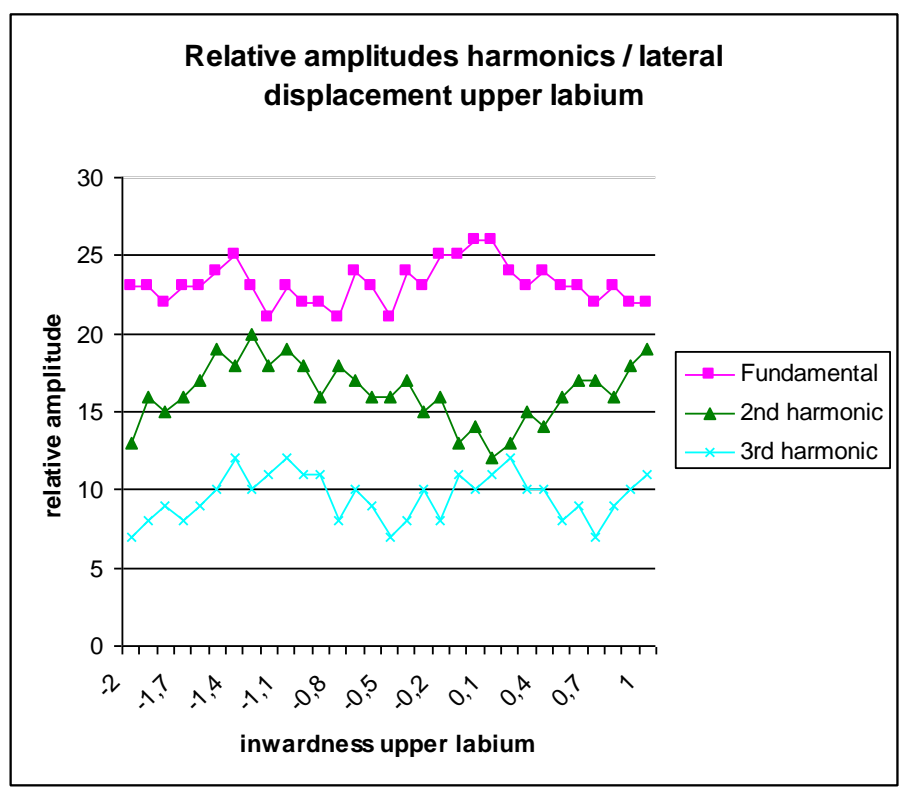

The second harmonic has a minimum near $0,3 \mathrm{~mm}$, while the third harmonic is minimum at two points around the symmetry axis.

\section{VOICING RULES}

Relatively little has been written on the subject of organ pipe voicing [Dom Bedos 1766, Goebel 1975, Monette 1992, Ellerhorst 1936, Bruder 1829, Pento 1995, Toepfer 1888, Cavaille-Coll 1840], and studying this material is not a good start into learning to voice. Most of these texts seem to have been copied from a notebook in the workshop on which the progress of the voicing process was logged. Even today the craft is transmitted on a one-to-one exchange basis from master to pupil (the artistic part is, for some obviously, not transmitted).

Some reports simply refrain to a simple list of causes and effects, such the following extract [Janke 2005]

\section{Niedriger Winddruck, weiter Fuß, weite Spalte lockere, schluchzende Ansprache voller Ton, klar 157. Hoher Winddruck, enger Fuß, enge Spalte zähe , fauchende Ansprache magerer Ton, matt} 158. Sehr dicker Kern, $85^{\circ}$ Fase, weite Spalte voller Ton mit gambigen Strich dumpfes Rauschen

Most authors use literary quite sophisticated phrases to express their ideas, such as "The windsheet either gets in its own way or gives out" [Monette 1992], when describing the air jet in action.

The only formula on voicing that ever seems to have been published is the 'Voicing Number' [Ising 1971]:

$$
I=\frac{V \cdot \sqrt{W}}{f \cdot H \cdot \sqrt{H}}
$$

where:

$I=$ the voicing number 


$$
\begin{aligned}
& \mathrm{V}=\text { the maximum jet velocity } \\
& \mathrm{W}=\text { the flue width } \\
& \mathrm{H}=\text { the cut-up }
\end{aligned}
$$

When I=2 the pipe is supposed to operate with maximum power. When I lies between 2 and 3 the pipe becomes gradually more principallike. Above $\mathrm{I}=3$ the pipe overblows.

Its derivation using dimensionless analysis is obscure and it shows some anomalies. When $\mathrm{I}=2$, for a pipe sounding optimally, one can expect $\mathrm{V} /(\mathrm{fH})$ to be 4 as shown before. This would imply a $\mathrm{W} / \mathrm{H}$ ratio of $1 / 4$ which corresponds to the geometry of a recorder but not of an organ pipe. The tendencies predicted for rising I are reasonable but, all other thing being equal, $\mathrm{V}$ can not produce the same effects as $\sqrt{\mathrm{W}}$. The number never seems to have been used very much anyway.

An exhaustive review of writings on voicing will be the subject of a future paper. The question will be raised whether to what degree these explanations correspond to real voicing practice and whether the results of it can be traced in organs still in existence.

\section{AN AERODYNAMIC APPROACH TO VOICING METHODS}

As the sound of flue instruments usually is quite distinctive from other wind instruments, it is clear that the air jet and its interaction with the air column plays a major role. They are mainly characterized by:

jet velocity profile, depending on flue channel geometry and varying along the jet. This parameter can be roughly characterized by a jet width and a maximum velocity. Practically, for a given flue geometry, it is mainly controlled by toe hole diameter and flue width. Roughly, since these parameters determine the amount of air supplied into the pipe and thus with the energy delivered to the pipe, they tend to be the main parameters, but certainly not only or the only ones, to determine the loudness.

upper labium location, which strongly influences the way the jet interacts with the acoustic flow. This parameter is usually divided into distance from the flue along the jet centreline ('cut-up') and lateral displacement with respect to this centreline. Practically, both of these parameters can be adjusted, languid lowering or heightening having a rather similar effect as the latter. These parameters, since they influence the air jet flow modulation, will tend to have more effect on the spectrum of the oscillating flows.

\section{1. $\quad$ Air jet velocity}

The air jet velocity is of major importance to the loudness and the stability regime of the pipe, it mainly depends on the toe hole cross section. The toe hole is not part of the oscillating system, it merely feeds a small stagnation chamber in the foot of each pipe which in turn supplies air to the flue. The shape of the toe hole can lead to turbulences in the flow which could propagate well into the air jet. Used as a flow constriction it can reduce the pressure in the wind chamber and thus the air jet velocity. This leads to a first family of voicing practices traditionally based on toe hole regulation, and practiced mainly in the XVIIIth and XIXth century but known to and used by organ builders long before and after this period.

By keeping the flue width at a constant large value in these methods the air flow injected by the air jet is thus mainly determined by its velocity. Accordingly a bright and full sound can be obtained through a relatively low cut-up, but strongly increasing the loudness by opening the toe hole also requires increasing the cut-up, thus making the sound less bright.

Looking for a dimensionless parameter to characterize this voicing family, a natural choice seems to be:

$$
S t r_{H}=f H / V
$$

It expresses the fraction of jet transit time through the mouth to the oscillation period, the parameter is given this specific name because formally it belongs to the socalled Strouhal numbers, which in general relate the time en space scales of a process to some characteristic velocity. Voicing with $\mathrm{Str}_{H}$ lower than the standard value $1 / 4$ gives overblown regimes which are very stable, and vice versa. How it can characterize loudness will be the object of further analysis and measurements.

\subsection{Jet width}

While the maximum velocity of the air jet is mainly determined by the pressure in the wind chamber below the flue, the air jet width depends entirely on the flue width and the geometry of the wind channel leading to 
the flue. This leads to a second family of voicing methods where the air jet flow is determined by the latter parameter, the pressure being kept constant by making the toe hole wide. Consequently the cut-up tends to be rather high and the slender high aspect ratio air jet turns out to stimulate well the higher harmonics. This voicing method seems to have been preferred in the XVIlth century during the heydays of Baroque organ building, when builders tried to obtain as much loudness as possible with as little wind consumption as possible. Using a low wind pressure it is possible to reduce the cut-up and thus delicate and transparent sounds ideally suited for polyphonic music are obtained. Increasing loudness by opening the flue will slightly brighten the timbre [Monette 1992, Janke 2005].

A good candidate to characterize this family is the air jet aspect ratio $\mathrm{W} / \mathrm{H}$, which strongly determines how the jet develops and thus interacts with the cross-flow. The linear model for the jet displacement breaks down for large aspect ratio values [Dequand 2001], in which a vortex model, consisting of vortices developing alternatively on both sides [Holger et al 1977] becomes more realistic. Further measurements are needed to determine the applicability limits and corresponding aspect ratio value for the case of open toe voicing.

\subsection{Mouth end correction}

The air jet transit time largely determines the self-sustained oscillation regime, in that it determines what the operating frequency will be with respect to the first passive pipe resonance. As seen before the mouth end correction has a considerable influence on the resonator: it can strongly influence the relative position of the operating frequency harmonics to the passive resonances. Accordingly, it can give rise to a specific voicing family where the characteristics of the mouth combine to give an aerodynamic control parameter. The geometry of the mouth is mainly defined by the cut-up, the mouth width, the presence of ears and other provisions to stabilize the air jet. Cut-up is the primary regulation parameter. A high cut-up can accommodate larger phase delays on the air jet and thus the operating frequency will be lower than the first pipe resonance, which will bring the higher harmonics further out of tune with the higher pipe resonances. The result is a decrease in harmonic content, the sound becomes duller. According to this behaviour some voicing methods start with pre-determined cut-up values and adjust the air jet parameters in order to obtain an appropriate loudness-brightness balance. Low cut-up is invariably associated with a thin and bright spectrum with a weak fundamental, whereas high cut-up brings about a broad, not necessarily very interesting, sound [Pento 1995].

The way the end correction voicing, especially of the mouth, influences the pipe resonances can be characterized by a dimensionless parameter $L_{m} / L$. For narrow-scaled pipes this value will be, considering (1) and typical proportions of the mouth, of order 0.05 , corresponding to the soft violinlike sound of this socalled string family. The other extreme two orders of magnitude larger is reached with the broad-scaled dull sounding flute pipes, where everything contributes to maximum inharmonicity.

\subsection{Air jet lateral displacement relative to upper labium}

This regulation has a major impact on the sound spectrum of the speaking pipe, yet is almost completely out of direct observation: minute alterations of the languid height with respect to the lower labium can cause strong displacements of the air jet with respect to the upper labium. Moreover the non-linearity introduced by the air jet flow dividing effect of the upper labium is the main source of harmonic generation in the pipe, but apparently the elusive character of this parameter has precluded it from becoming a basic regulation parameter of some voicing method. Rather it is actively used as a welcome additional degree of freedom capable of strongly influencing harmonic development and transient behaviour.

As a characteristic parameter the ratio $\mathrm{y}_{0} / \mathrm{H}$, where $\mathrm{y}_{0}$ is the lateral displacement of the jet axis with respect to the upper labium, could be useful. Further study is needed to determine the strength of this.

\subsection{The optimal operating point}

Several authors mention the existence of regions of voicing parameter values which allow 'optimal' operation of the pipe or part of it.

\subsubsection{The air jet}

Numerical studies of jet instability [Drazin\&Howard 1966] show that the growth coefficient $\mu$ is not constant along the developing jet but shows a maximum where the jet thickness equals one fifth of the disturbance wavelength. Roughly assuming the air jet to spread with $12^{\circ}$ so that the jet thickness is about $2 / 5$ of the distance covered, this will take place at a distance of half a disturbance wavelength. This is precisely the condition of a phase delay on the jet corresponding to the pipe sounding at her first passive resonance frequency of the air column and thus a lucky coincidence. 
Moreover the acoustic field around the pipe mouth turns out to have a non-uniform velocity distribution across the mouth. The next figure shows a simulation of the acoustic (potential) flow field in the pipe mouth, using some geometric simplifications [Verge 1995]:

Velocities much higher than average are found near the labium, in the region of large $\mu$. The result is that the air jet suddenly becomes strong lateral displacements as it approaches the upper labium, as shown in the following Schlieren visualisation, showing different phases of one period of steady oscillation (left to right, right is inside the pipe):

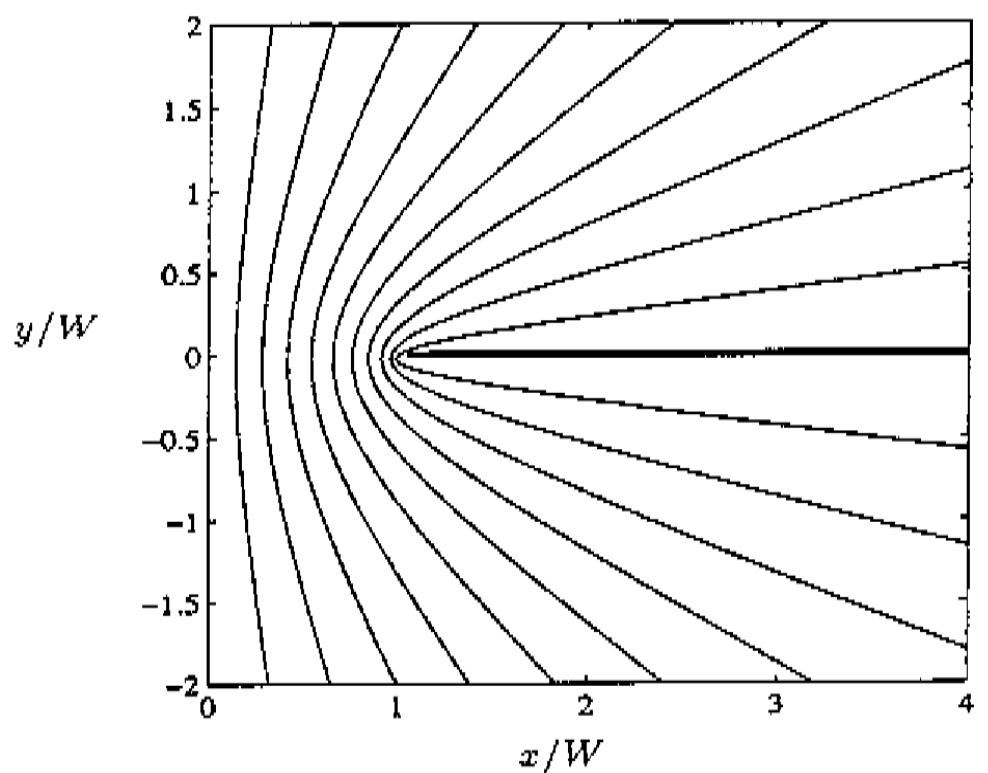

$x / W$
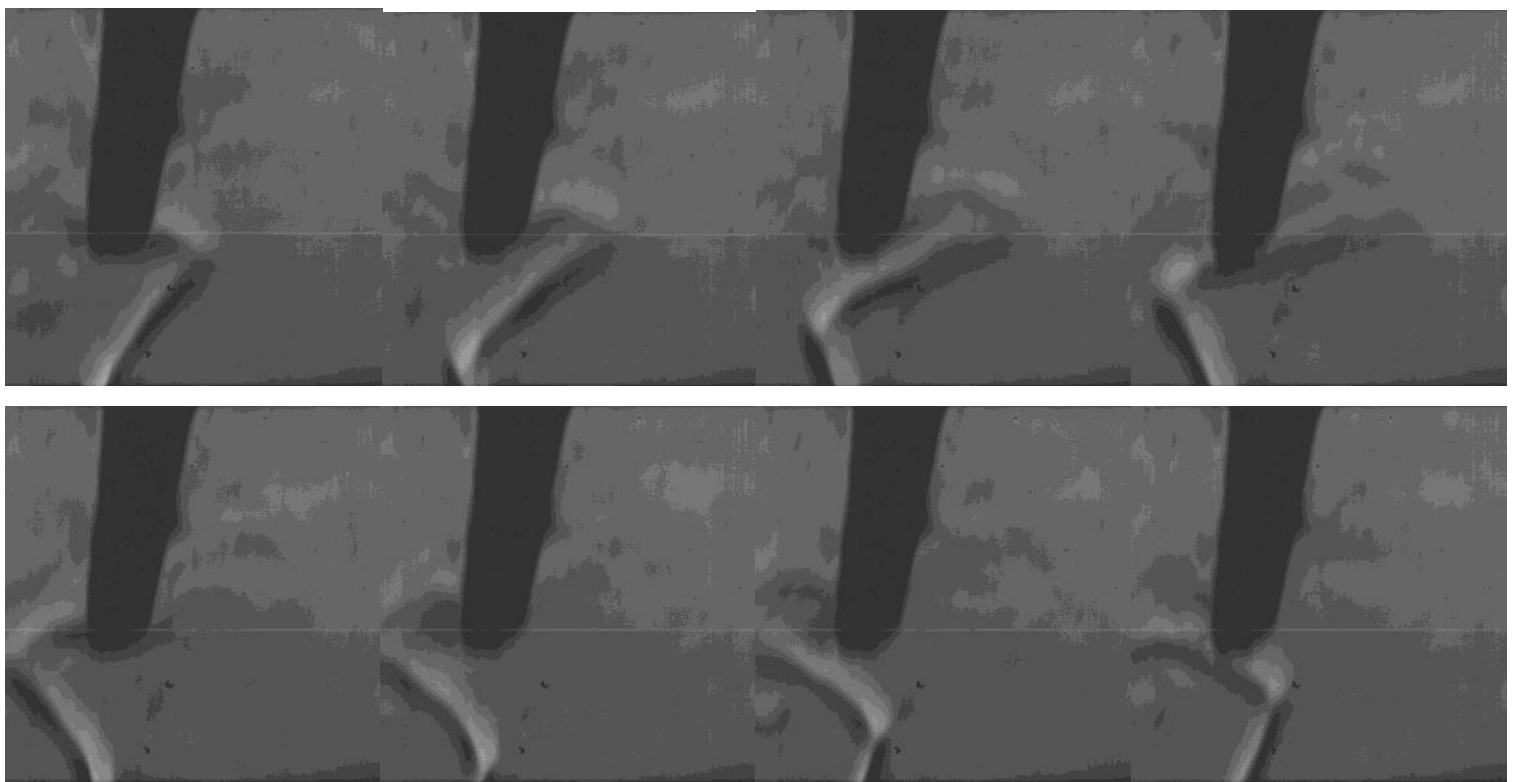

It is clearly visible that in the first half of the period the upper part of the jet is convecting into the pipe and this all the faster the more the upper labium is approached because of the larger acoustic velocity field, As a result the upper part of the jet is more and more tilted. At the end of the first half of the period the acoustic field velocity changes direction and process now repeats in the other direction. Details on the operating conditions for these images are found in [Steenbrugge 2009].

From these qualitative data it can also be seen that the air jet flow starts to enter the pipe when the acoustic flow into the mouth is maximum, thus confirming the phase delay of around $1 / 4$ of a period previously mentioned for a pipe sounding at its passive resonance frequency.

It can be hypothesized that the perfectly periodic oscillations which flue instruments are able to generate, despite the fundamentally unstable nature of the source medium, is essentially due to the presence and cooperation at the same location of these three fluid dynamical phenomena: acoustic flow concentration, excessive air jet instability and optimal disturbance phase conditions on the jet. 


\subsubsection{Toe hole and flue width voicing}

A short experimental analysis [Nolle 1979] concluded that toe hole and flue width voicing can be interchanged within a certain range of parameters. Accordingly the acoustic pressure was found to be proportional to the product of wind pressure and flue width and harmonic content was constant as far as the first 3 or 4 harmonics were concerned. This suggests that toe hole voicing and flue width voicing are extreme cases that evolve toward each other when the respective basic parameters are being mixed. These observations and their relationship to the other optima discussed in this paper will be the subject of further study and measurement.

\section{PHYSICAL MODELLING AND VOICING}

The number of applications of physical modelling of systems not developed from physics first principles, such as natural phenomena or art objects, has greatly increased with the introduction of more and more powerful data processing capabilities. Accordingly virtual representations of musical instruments has become a wide research topic, but the field has always faced a formidable challenge: acoustical instruments, and especially wind instruments, usually and necessarily have an internal life which is very different from their external appearance. By the laws of acoustics the low frequency end of the oscillations is almost unable to escape from it, whereas the high frequency part is quite freely liberated. This means that the instrument essentially operates through the lowest frequency components, which, taking into account their sequestration, must be very strong, and the high frequencies, the ones evolution has brought living beings to be most interested in, are in fact nothing more than weak superfluous byproducts. Internally the high frequency part of the instrument's operation is thus a higher order effect whereas most current physical models are limited to lower order behaviour.

Practical applications of physical modelling, for instance in sound synthesis, therefore usually require the use of fine tuning parameters, the effect of which is not explicitly built into the model but is controlled by an external and adequately sensible agent. This is the situation in the mainstream application of musical instruments modelling which consists in creating virtual instruments.

As suggested above, physical modelling of organ pipes not only allows to disentangle voicing practices and gain a better understanding of efficient voicing methods, it can be a useful toolbox with which the most important aspects of a specific organ builder's voicing practices can be characterized. As a small demonstration, the late organ builder Charles Fisk, who introduced neo-baroque organs in the USA during the 1960's, writes:

"If you first agree that the windway must be kept open (almost the width of the material of the lower lip) ..., then classical voicing is a matter of balancing the toe hole opening and the cutup (i.e., the height of the mouth opening). If we choose a wide open toe hole, then underblowing will be achieved if we raise the cutup just beyond the point at which the pipe appears to be giving out its maximum volume of sound. Thus the classically voiced organ pipe is one in which the cutup is "a little too high." With the cutup on the high side, the tone becomes fuller and gentler; more important, the pipe is not quite as stable as it would be with a lower cutup, and this makes it much more easily influenced by pulses or irregularities in the flow of air coming through its toe hole." [Fisk 1976]

This recipe, which essentially belongs to the toe hole regulation family, can be readily understood and memorized if one is reminded of the 'optimal' cut-up distance where $1 / 2$ of a disturbance wavelength is on the air jet. This is Fisk's point of 'maximum volume of sound'. Raising the cut-up while leaving the toe hole and thus pressure the same underblows the pipe, and the jet velocity profile near the upper labium has become smoother whence less harmonic drive. On the other hand, increasing the mouth surface increases mode competition because the higher passive resonances become more harmonic: the pipe looses some of its stability. Explaining Fisk's voicing rule has required no other knowledge than what was presented above in the short overview of flue instrument models.

Arrived at this point however the biggest problem remains, which is the determination of voicing techniques used in extant organs, especially if little or no information is available from or about the builder/voicer. As the voicing parameters are not mutually independent in their effect it is usually difficult to conclude about a specific voicing method used on the basis of these parameters alone, except in extreme cases. What is needed is what the taxonomist used to do when a new species was discovered, a comparison is made between the specimen and similar ones in a collection. As the history of voicing methods and their inheritance has not yet been written this identification task is very difficult. Moreover the question arises which data such data bases need to contain in order to seriously allow this comparison. It is well known that even the most complete inventories of organ pipes do not contain all necessary values needed to be able to faithfully reproduce a given pipe with a given sound. For instance the angle of attack of the air jet, so important for the acoustic properties, is never included because it's not part of the geometry. Toe hole dimensions are often given but their cross-section is often so irregular that a simple description is 
inaccurate because a small variation can cause large pressure differences in the pipe foot. A first step towards a better documentation would certainly be a quantitative description of the all-important velocity profile along the jet: its amplitude, its lateral and longitudinal velocity distribution, its direction, its width, etc...

\section{CONCLUSION}

Based on the aerodynamic properties of the self-sustained oscillations in the flue organ pipe, different voicing categories were identified and briefly characterized. Because they are not directly based on geometrical parameters it's easier to describe cause and effects in each of them in terms of mutually independent dimensionless quantities. The relevancy of the chosen quantities, which have been chosen largely on the basis of good guesswork, has to be further established by measurements on more different cases.

From the presented short overview of the aerodynamics in flue instruments it became clear that the air jet is the essential operator in the system: it is the active element allowing positive feedback, it accommodates in a rather flexible way necessary phase delays, and together with the upper labium it constitutes the main source of inharmonicity which is of the highest relevance from a musical point of view. The air jet behaviour is very sensitive to small geometric variations in the mouth and therefore analyzing the voicing of flue pipes could benefit from taking into consideration the aerodynamics in the mouth region.

Further work in this direction will include trying to more exhaustively and quantitatively characterize different voicing styles by means of the aerodynamic parameters presented in this paper.

\section{ACKNOWLEDGMENTS}

The authors would like to acknowledge the financial support of the Onderzoeksfonds HoGent.

\section{REFERENCES}

[1] Audsley G. A.., The Art of Organ Building 2Vol. Dover Publications 1905/1965.

[2] Cavaillé-Coll, A. (1840). Études Expérimentales sur les Tuyaux d'Orgues. In Complete Theoretical Works of A. Cavaillé-Coll. Buren (GLD.), The Netherlands: Frits Knuf 1979

[3] Bédos de Celles (Dom), F. (1766-78). L'art du facteur d'orgues - 4 vols.; facsimile edition, Paris: Bärenreiter-Kassel, 1958.

[4] Bruder I., Orgel- und Spieluhrenbau, Aufzeichnungen des Orgel- und Musikwerkmachers Ignaz Bruder aus Waldkirch von 1829 und die Entwicklung der Walzenorgel Vierunddreissigste Veröffentlichung der GdO 1968 by Sanssouci Verlag AG Zürich,

[5] Dequand, S., Luo, X., Willems, J. F. H., and Hirschberg, A. 2001!. "Self- Sustained Oscillations in a Helmholtz-like Resonator. Part 1: Acoustical Measurements and Analytical Models," in 7th AIAA/CEAS Aeroacoustics

[6] Conference Maastricht, NI!, submitted to the AIAA Journal

[7] Drazin P.G. \& Howard L.N. Hydrodynamic stability of parallel flow of inviscid fluid, Adv. Appl. Mech. 9:1-89, 1966.

[8] Ellerhorst W.: Handbuch der Orgelkunde. Einsiedeln 1936, Frits Knuf, Buren 1986 (3. Repr.), ISBN 90-6027-519-5

[9] Fabre, B., and Hirschberg, A. 2000!. "Physical Modelling of Flue Instruments: a Review of Lumped Models," Acust. Acta Acust. 86, 599-610.

[10] Fisk C., Articulateness and the Organ: A problem for the Organ Builder Lecture given at the dedication of the Flentrop Organ, Duke University, November 1976

[11] Fletcher, N. H. \& Rossing, T. D. (2005). The Physics of Musical Instruments. New York: SpringerVerlag

[12] Fletcher N.H.(2001), Recent progress in the acoustics of wind instruments, Acoust. Sci. \& Tech. 22, 3.

[13] Goebel, J. (1975). Theorie und Praxis des Orgelpfeiffenklanges. Frankfurt am Main.

[14] Holger, D. K., Wilson, T. A., and Beavers, G. S. 1977!. "Fluid mechanics of the edge-tone," J. Acoust. Soc. Am. 62, 1116-1128.

[15] Ingerslev F. \& Frobenius W.(1974), Some measurements of the end-correction and acoustic spectra of cylindricalopen flue organ pipes. Tran. Dan. Acad. Tech. Sci. 1, 1-44.

[16] Ising, H. (1971). "Erforschung und Planung des Orgelklanges." Walcker Hausmitteilung, 42, 38-57.

[17] Janke R, (2005) Intonationsvorschriften, 
Day of Research 2010 - February 10 - Labo Soete, Ghent University, Belgium

[18] Levine H. \& Schwinger, J. (1948), On the radiation of sound from un unflanged circular pipe, Phys. Rev. 74, 383-406.

[19] M. E. Mclntyre, R. T. Schumacher and J. Woodhouse. (1983), On the oscillations of musical instruments. J. Acoustical Society of America 74 1325-1345.

[20] Monette, L. G. (1992). The Art of Organ Voicing. Kalamazoo, MI: Western Michigan University

[21] Nolle A., Sinuous instability of a planar air jet: Propagation parameters and acoustic excitation, J. Acoust. Soc. Am. 103(6), 3690-3705.

[22] Pelto, P. (1995). "Four Voicing Techniques," The Organ Yearbook (Vol. XXV).

[23] Pierce A.D., Acoustics. McGraw-Hill, NY, Acoustical Society of America 1990.

[24] Sato H. ,The Stability and Transition of a two-dimensional Jet, J.Fluid.Mech.7, 53-80. 1937

[25] Ségoufin, C., Fabre, B., Verge, M. P., Hischberg, A., and Wijnands, A. P. J. (2000). "Experimental Study of the Influence of the Mouth Geometry on Sound Production in a Recorder-like Instrument: Windway Length and Chamfers." Acustica, 86(4), 649-661.

[26] Steenbrugge (2009) "A flue organ pipe with micro-adjustable mouth geometry : design and measurement procedures". Hogeschool Gent.

[27] Töpfer J.G., „Die Theorie und Praxis des Orgelbaues“ [Buren] : Knuf. 1888/1972

[28] Verge, M.-P. (1995). Aeroacoustics of Confined Jets with Applications to the Physical Modeling of Recorder-Like Instruments. Techische Universiteit, Eindhoven 